\title{
Management of Patients with Interstitial Lung Disease in the Midst of the COVID-19 Pandemic
}

\author{
Katerina M. Antoniou ${ }^{a}$ Ganesh Raghu ${ }^{b}$ Vasilios Tzilas ${ }^{c}$ Demosthenes Bouros ${ }^{c}$ \\ a Laboratory of Molecular and Cellular Pneumonology, Department of Respiratory Medicine, University General \\ Hospital of Heraklion, Medical School, University of Crete, Heraklion, Greece; ${ }^{b}$ Division of Pulmonary, Sleep and \\ Critical Care Medicine, University of Washington, Seattle, WA, USA; ${ }^{C}$ Athens Medical Center and 1st Department of \\ Respiratory Medicine, "Sotiria" Hospital for Diseases of the Chest, National and Kapodistrian University of Athens, \\ Athens, Greece
}

Interstitial lung diseases (ILDs) comprise a heterogeneous group of acute and chronic lung diseases that cause progressive scarring of the lung tissue, compromising respiratory function and blood oxygenation. The most common form is the idiopathic pulmonary fibrosis (IPF), which in the vast majority of cases affects the older population, in a progressive fibrosing manner, resulting in severe respiratory failure and death within 3-5 years [1]. Public health officials recommend that patients in the higher risk category should reduce the risk of being exposed to SARS-CoV-2 $[2,3]$. Patients with IPF, sarcoidosis [4], and other ILDs with known etiology, such as rheumatologic diseases with ILD involvement, could be at particular risk for SARS-CoV-2, since they tend to be older, have multiple comorbidities, and are often immunosuppressed by their disease or therapy [5]. Currently, there is no data regarding the incidence of COVID-19 in the field of ILDs.

One of today's challenges for physicians treating ILD patients is how to protect them from and treat them for COVID-19. If they are mildly symptomatic or non-symptomatic, can they return home safely, or may they deteriorate and will need hospitalization? Besides, it is important to differentiate the manifestation of superimposed COVID-19 from progression of their underlying fibrotic

karger@karger.com

(c) 2020 S. Karger AG, Basel

www.karger.com/res

Karger
ILD and/or acute exacerbation known to occur within the spectrum of their underlying ILD. These questions are crucial for patient care.

Furthermore, patients are deprived of pulmonary rehabilitation. Routine follow-up, face-to-face visits are made via telephone or are rescheduled, oral medications are delivered to the patient's home to cover the peak period of the pandemic, and biological samples are collected and processed at a local facility near to the patient's residence. The multidisciplinary discussions are done via a virtual platform, and treatment decisions are made with suboptimal objective assessment over the telephone during this period of COVID-19. However, the aforementioned necessitated steps of actions for the diagnosis and management of these patients with ILD have been postponed due to restrictions in some regions and countries burdened with COVID-19 patients. More in detail, the COVID-19 pandemic directly affect patients with fibrotic ILD in multiple ways. There are major issues to access key components of the diagnostic process, uncertainties in the use of common ILD therapies, and there is limited ability to monitor both disease severity and the presence of medication adverse effects, which are extensively discussed in a recent review [6]. 
In the midst of so much uncertainty in the status of the COVID-19 pandemic, the abrupt changes in global health have surfaced several clinical and scientific questions and concerns for the ILD community at large. This includes a serious and disruptive effect on the conduct of ILD clinical trials, with short- and long-term consequences. While screening and recruitment for the enrollment of patients in ongoing clinical trials have been on hold indefinitely, the safety of patients enrolled and participating in clinical trials is being assured with modified protocols that include home visits by nurses and/or telemedicine to monitor clinical status. Moreover, hospital radiology departments and laboratories are stretched during the pandemic, and to reduce the risk of SARS-CoV-2 infection for trial and ILD subjects, as with other chronic respiratory diseases, some protocol-mandated visits and study procedures as bronchoscopy assessments with biopsies and bronchoalveolar lavage fluid have been delayed or cancelled all over the world.

Several media reported cases of pulmonary fibrosis resulting from COVID-19 disease, which raised important questions in the pulmonary fibrosis community. Most importantly, acute exacerbation leads to an in-hospital mortality of more than $50 \%$ with a mean survival time of only a few months [7]. Therefore, consideration of complications in IPF is of great importance for the understanding of the disease and treatment planning. Patients who are infected with COVID-19 may develop acute exacerbation such as pneumonia and progress to respiratory failure and acute respiratory distress syndrome (ARDS), which requires life support with a mechanical ventilator. The cardinal role of vaccination in this population has to be emphasized; the potential of lung-directed immunotherapy to induce an antifibrotic $\mathrm{T}$-cell response capable of arresting and reversing lung fibrosis is interesting [8].

In a subset of COVID-19 SARS survivors, post-COVID-19 lung fibrosis will need to be addressed. Post-SARS fibrosis and pulmonary fibrosis that occurs in ILDs have distinct differences. Post-SARS fibrosis typically is not progressive, but nonetheless can be severe and limiting. The recovery period for post-SARS fibrosis is approximately 1 year, and the residual deficits persist but generally do not progress [9]. Increased collagens and abnormal extracellular matrix components in the lungs of patients with ARDS and IPF compared to normal human lungs have been demonstrated [10], and it is unknown if the genetic susceptibility to manifest fibroproliferative response in the lungs of patients with ARDS, SARS, IPF, and other fibrotic ILDs are similar. However, the avail- able data so far are limited, since histopathology features in the lungs from patients with COVID-19 have been described in a few patients at autopsy [11]. Postmortem biopsies were performed at Lille University on 6 COVID19 -positive patients at different stages of the disease. For 5 out of 6 patients who died later in their disease course [11], the histologic pattern was an acute fibrinous and organizing pneumonia (AFOP), characterized by an extensive intra-alveolar fibrin deposition so-called fibrin «balls», rather than hyaline membranes. According the aforementioned results, understanding the correct pathophysiology is crucial to establishing the basis for appropriate treatment, both in terms of mechanical ventilation and further follow-up and possible management with antifibrotic drugs in the progressive phenotypes [12]. The latter scenario also seems safer, as it is rather risky for the patient to initiate immunomodulation in the pandemic era. The possibility of shared mechanisms of fibrosis between ARDS cases and chronic ILDs raises the potential that therapies that treat ILDs could also be beneficial to COVID-19 associated lung disease $[13,14]$. In this regard, pirfenidone is being used in patients with COVID-19 in Wuhan, China (clinical trial.gov), and a prospective clinical trial with the other antifibrotic drug, nintedanib, is discussed [13], taken in consideration the shared pathogenetic and clinical similarities of COVID-19 and the fibrotic process. We share the opinion that at present, the long-term pulmonary consequences of COVID-19 remain speculative and should not be assumed without appropriate prospective studies [14]. Furthermore, we need to be prepared for providing care and monitor the interstitial pneumonia/fibrotic ILD in survivors of COVID-19 and associated SARS. It is hoped that an international task force with experts will be able to provide guidance to the community at large to manage the new disease COVID-19-ILD.

\section{Conflict of Interest Statement}

The authors declare that they have no conflicts of interest to disclose.

References

1 Raghu G, Remy-Jardin M, Myers JL, Richeldi L, Ryerson CJ, Lederer DJ, et al.; American Thoracic Society, European Respiratory Society, Japanese Respiratory Society, and Latin American Thoracic Society. Diagnosis of Idiopathic Pulmonary Fibrosis. An Official ATS/ERS/JRS/ALAT Clinical Practice Guideline. Am J Respir Crit Care Med. 2018 Sep; 198(5):e44-68. 
2 British Thoracic Society Advice for Managing Interstitial Lung Disease Patients during COVID-19 pandemic. Available from: https:// brit-thoracic.org.uk/media/455101/bts-management-advice-for-ild-patients-v1023-march-2020.pdf.

3 Halpin DM, Faner R, Sibila O, Badia JR, Agusti A. Do chronic respiratory diseases or their treatment affect the risk of SARS-CoV-2 infection? Lancet Respir Med. 2020 May;8(5): 436-8.

4 Sweiss NJ, Korsten P, Syed HJ, Syed A, Baughman RP, Yee AM, et al. When the Game Changes: Guidance to Adjust Sarcoidosis Management During the COVID-19 Pandemic. Chest. 2020 Apr:S0012-3692(20)30778-9.

5 Ceribelli A, Motta F, De Santis M, Ansari AA, Ridgway WM, Gershwin ME, et al. Recommendations for coronavirus infection in rheumatic diseases treated with biologic therapy. J Autoimmun. 2020 May; 109:102442.

6 Wong AW, Fidler L, Marcoux V, Johannson KA, Assayag D, Fisher JH, et al. Practical Considerations for the Diagnosis and Treatment of Fibrotic Interstitial Lung Disease During the COVID-19 Pandemic. Chest. 2020 Apr;S0012-3692(20)30756-X.

7 Kreuter M, Polke M, Walsh SL, Krisam J, Collard HR, Chaudhuri N, et al. Acute exacerbation of idiopathic pulmonary fibrosis: international survey and call for harmonisation. Eur Respir J. 2020 Apr;55(4):1901760.

8 Collins SL, Chan-Li Y, Oh M, Vigeland CL, Limjunyawong N, Mitzner W, et al. Vaccinia vaccine-based immunotherapy arrests and reverses established pulmonary fibrosis. JCI Insight. 2016 Apr;1(4):e83116.

9 Hsieh MJ, Lee WC, Cho HY, Wu MF, Hu HC, Kao KC, et al. Recovery of pulmonary functions, exercise capacity, and quality of life after pulmonary rehabilitation in survivors of ARDS due to severe influenza A (H1N1) pneumonitis. Influenza Other Respir Viruses. 2018 Sep;12(5):643-8.

10 Raghu G, Striker LJ, Hudson LD, Striker GE. Extracellular matrix in normal and fibrotic human lungs. Am Rev Respir Dis. 1985 Feb; 131(2):281-9.
11 Copin MC, Parmentier E, Duburcq T, Poissy J, Mathieu D; Lille COVID-19 ICU and Anatomopathology Group. Time to consider histologic pattern of lung injury to treat critically ill patients with COVID-19 infection. Intensive Care Med. 2020 Jun;46(6):1124-6.

12 Gattinoni L, Chiumello D, Caironi P, Busana M, Romitti F, Brazzi L, et al. COVID-19 pneumonia: different respiratory treatments for different phenotypes? Intensive Care Med. 2020 Jun;46(6):1099-102.

13 Spagnolo P, Balestro E, Aliberti S, Cocconcelli E, Biondini D, Della Casa G, et al. Pulmonary fibrosis secondary to COVID-19: a call to arms? Lancet Respir Med. 2020 Aug; 8(8):P750-52. Available from: https://doi. org/https://doi.org/10.1016/S22132600(20)30222-8.

14 George PM, Wells AU, Jenkins RG. Pulmonary fibrosis and COVID-19: the potential role for antifibrotic therapy. Lancet Respir Med. 2020 Aug;8(8):P507-15. Available from:https://doi.org/https://doi.org/10.1016/ S2213-2600(20)30225-3. 